\title{
Design of Heart Sounds and ECG Real-time Auscultation System Based on LabVIEW
}

\author{
Kunliang Xu \\ ${ }^{1}$ School Of Computer Science And Engineering of Qujing Normal University, \\ QuJing, 655011, China \\ xkunl_2006@163.com
}

\begin{abstract}
This paper introduces a designed Real-time auscultation system based on Labview which can conduct data acquisition and playback, using a PCI - 9101 data acquisition card to obtain the signal of heart sounds and ECG, cooperating with front-end signal to adjust circuit, can effectively extract the weak heart sounds, ECG signal, the front-end signal conditioning circuit takes a series of measures of the filtering, and adopting photoelectric isolated technology between the weak signal and the strong signal, ensuring the safety of patients in the process of auscultation. The system solves better the interference problem, providing better data quality for real-time auscultation, the use of the system can provide greater support for medical diagnosis and actual data analysis, at the same time, visualizing the abstract auscultation knowledge visualization in the auscultation teaching, which has a certain value of use.
\end{abstract}

Keywords: heart sounds signal; ECG (Electrocardiograph) signal; Real-time auscultation system; LabVIEW

\section{Introduction}

Heart disease is the floorboard of all sorts of heart disease, wide coverage, and with the enhancement and improvement of modern living standards, the incidence and mortality of cardiovascular disease is also more and more high [1-2], according to the statistics data shows that every year the number of human deaths caused by heart disease accounted for $30 \%$ of all deaths, especially in the European and American countries, $40 \%$ people of about the age of 40 suffer from heart diseases [3], there are about 100000 people suffering from congenital disease in Chinese annual births [4], thus, heart disease has become a frequently occurring and commonly-seen diseases harming human health. Heart sounds is a reflection of the heart and cardiovascular system of mechanical movement, heart sounds contains the heart of each part and the interaction between physiological and pathological information, and it is important physiological signal in human body, carrying the physiological and pathological information of human cardiovascular system [5].

Since Laennec invented a stethoscope, the medical community started to widely use stethoscope to listen to the patient's heart sounds to make analysis judgment [6]. Studies have shown that [2], the most sensitive frequency range of the human ear hearing is between $1000-3000 \mathrm{~Hz}$, the sensitivity of low frequency is poorer, listen to the voice of less than $20 \mathrm{~Hz}$ is more harder, So, the human ear to those who have the important meaning of low frequency and intensity of the smaller heart sound signals are often unable to distinguish between. Obviously, this diagnosis method of the stethoscope auscultation relies too much on the doctor's subjective experience and the resolution of the human ear, and it is interfered greatly by environment, 
having a certain degree of subjectivity and one-sidedness, and the analytical results has a close relationship with the doctor's experience and knowledge.

In this paper, a simple heart sound real-time acquisition and playback of auscultation system, can be a safe and effective acquisition heart sounds, ECG signal and real-time display, solving the interference problem well, The use of the system can provide greater support for medical diagnosis and actual data analysis, the same time, visualizing the abstract auscultation knowledge visualization in the auscultation teaching, which has a certain value of use.

\section{Introduction of LabVIEW}

LabVIEW (Laboratory Virtual Instrument Engineering Workbench), namely the Laboratory Virtual Instrument integration environment, is the U.S. NATIONAL Instrument company innovative software products, It combines a graphical programming method of high performance and flexibility, provides a standard graphical development environment, is currently the most widely used and fastest-growing, most powerful graphical software development environment [8-9].

LabVIEW with extensible function library and the library of generic programming system, not only can be used for general Windows desktop application design, but also provides for GPIB equipment control, VXI bus control, serial device control, and data analysis application module, display and storage, etc. Look from the operation mechanism, is not the traditional von neumann by executive way of computer architecture, it is a kind of a graphic control Flow Data structure of Data stream model (the Data Flow Mode). Data flow process design rules, only when it is all the input effective execution, and the output of the target, is effective only when its function fully. So, LabVIEW is connected in the block diagram of the data flow between controls the execution sequence, rather than the Sue text program is sequential, therefore, we can through mutual connection function block diagram of the rapid application development, neatly even can have multiple data channels synchronous operation [8-9].

\section{System Block Diagram}

The system consists of heart sounds ECG sensors, signal conditioning circuit, data acquisition card and computer application software of several parts. First of all, Mind ECG sensors will heart sounds ECG signal into electrical signal, then completed by signal conditioning circuit of signal amplification, photoelectric isolation, filter, trap, such as signal disposal, Computer through a data acquisition card for heart sounds ECG signal and real-time display, the system block diagram is shown in Figure 1.

Signal conditioning circuit

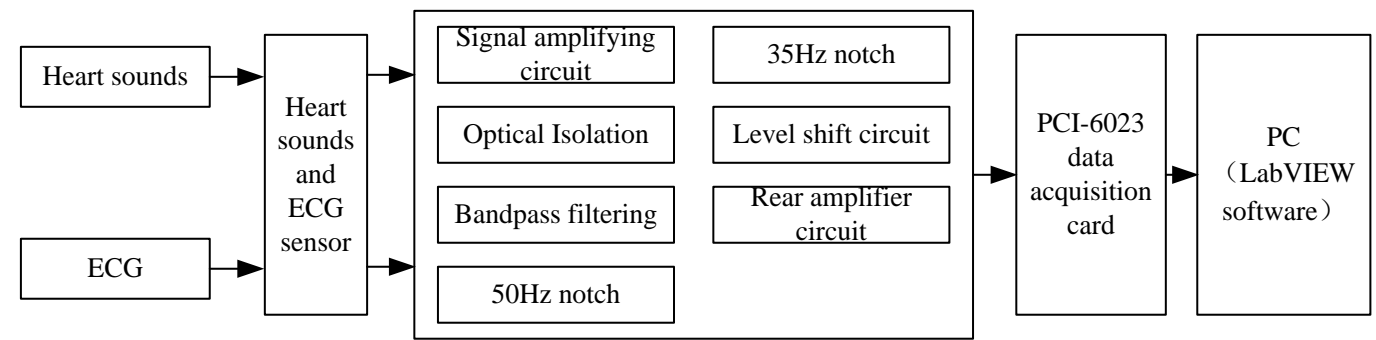

Figure 1. System Block Diagram 
In view of the heart sound signal frequency range is wide, the low frequency for about $3 \sim 5 \mathrm{hz}$, the high frequency about $600 \sim 800 \mathrm{hz}[10,14]$, trying to avoid outside interference signal in extracting weak heart sounds signals simultaneously, so we will be high sensitivity, strong anti-interference ability stethoscope modified for heart sound sensor. Due to a weak heart sounds is a mechanical vibration, the heart sound sensor received signal is very weak and mixed with low-frequency $(50 \mathrm{~Hz}$ frequency interference, $35 \mathrm{~Hz}$ or so human EMG interference, etc.), external high-frequency interference and other signals, to get a clean heart sounds signal, requiring high accuracy amplification, band-pass filter $(5 \sim 1500 \mathrm{~Hz})$, notch $(35,50 \mathrm{~Hz})$ and other circuits to filter out interfering signals. At the same time, we added a post-amplifier circuit, easy adjustment heart sound signal[11-13].

ECG is the frequency range of the human body is about $0.05 \sim 150 \mathrm{~Hz}$, by about 0 $\sim 4 \mathrm{mV}$ weak signal [14-15], the ECG signal picks up by a special electrode. ECG and heart sounds processing circuit similar to the circuit, only the band-pass filter in the range: $0.05 \sim 150 \mathrm{~Hz}$.

\section{The Main Hardware Circuit Structure}

\subsection{Preamplifier Circuit}

Preamplifier circuit is the key to heart sounds ECG data acquisition, the specific circuit shown in Figure 2, due to the relatively weak human heart sounds ECG, ECG and heart sounds are usually mixed with other biological signals, such as centralized around $35 \mathrm{~Hz}$ EMG human interference, $50 \mathrm{~Hz}$ power frequency interference, making ECG heart sounds strong background noise test conditions more complicated. For there is no distortion to detect the clinical value of clean ECG heart sounds, heart sounds are often required ECG acquisition system with high accuracy, high stability, high input impedance, high common mode rejection ratio, low noise and strong anti-interference performance.

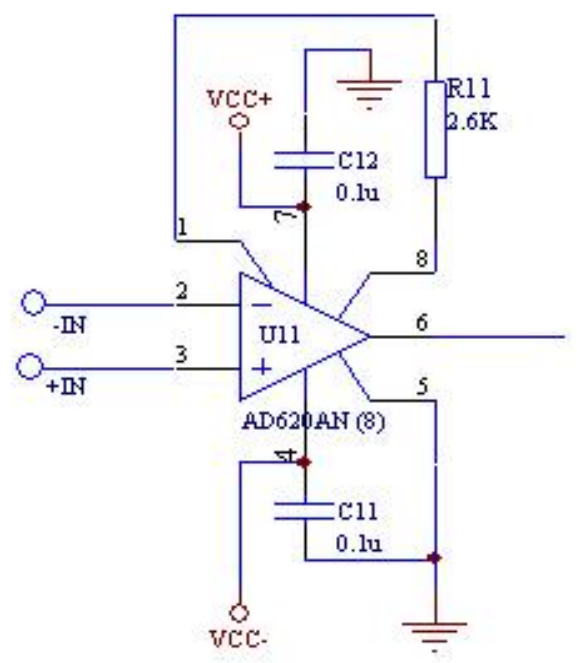

Figure 2. Preamplifier Amplification Circuit Diagram

This design uses AD's instrumentation amplifier AD620 as a pre-amplifier, AD620 inputs ultra- $\beta$ processing technology, with low input bias current, low noise, high-precision, high set-up time, low power consumption and other characteristics, common mode rejection ratio up to $130 \mathrm{~dB}$, very suitable for the use of medical 
instrumentation amplifier whose gain is adjustable (range of about 1 to 10,000 times) and by the formula

to determine [16-17].

$$
G=1+49.4 k \Omega / R_{g}
$$

\subsection{Band-pass Filter Circuit}

Band-pass filter circuit by a low-noise dual op amp NE5532 form. Fig. 3 shows the active low pass filter circuit, and Fig. 4 shows a combination of active high-pass filter circuit as a band-pass filter circuit. Fig.3, R42, R43, R44, R45, C42, C43, C44, C45 constitute cutoff frequency $f=5 \mathrm{kHz}$ fourth-order Butterworth low-pass filter.

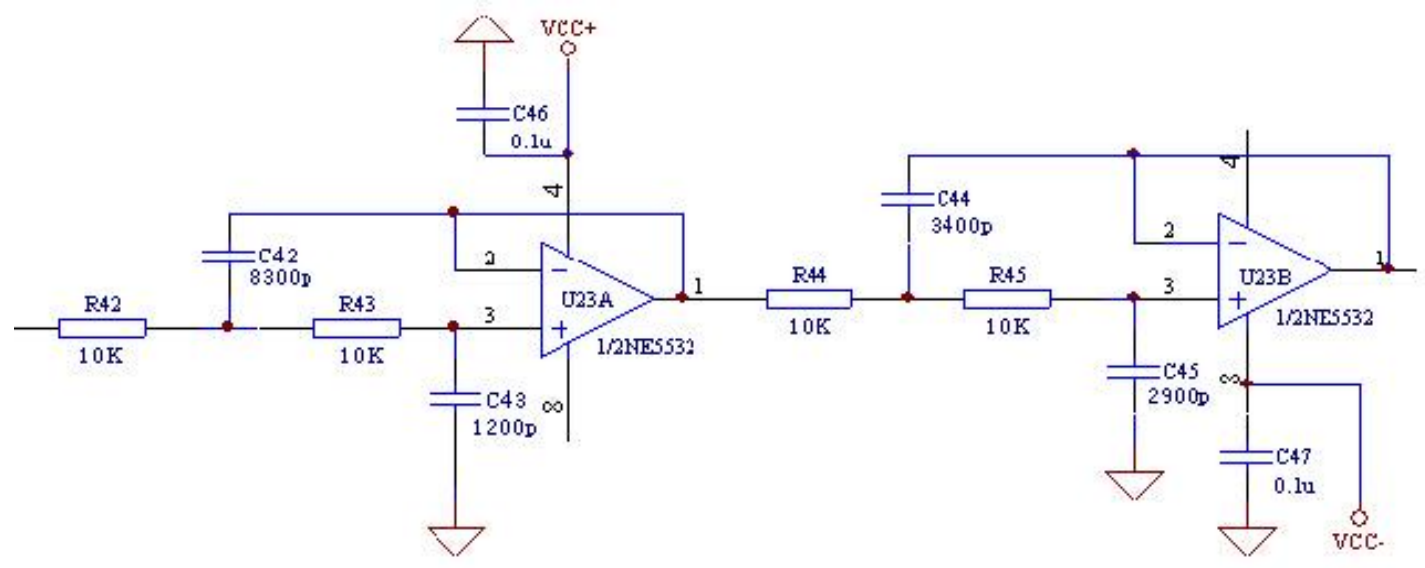

Figure 3. Low-pass Filter Circuit Diagram

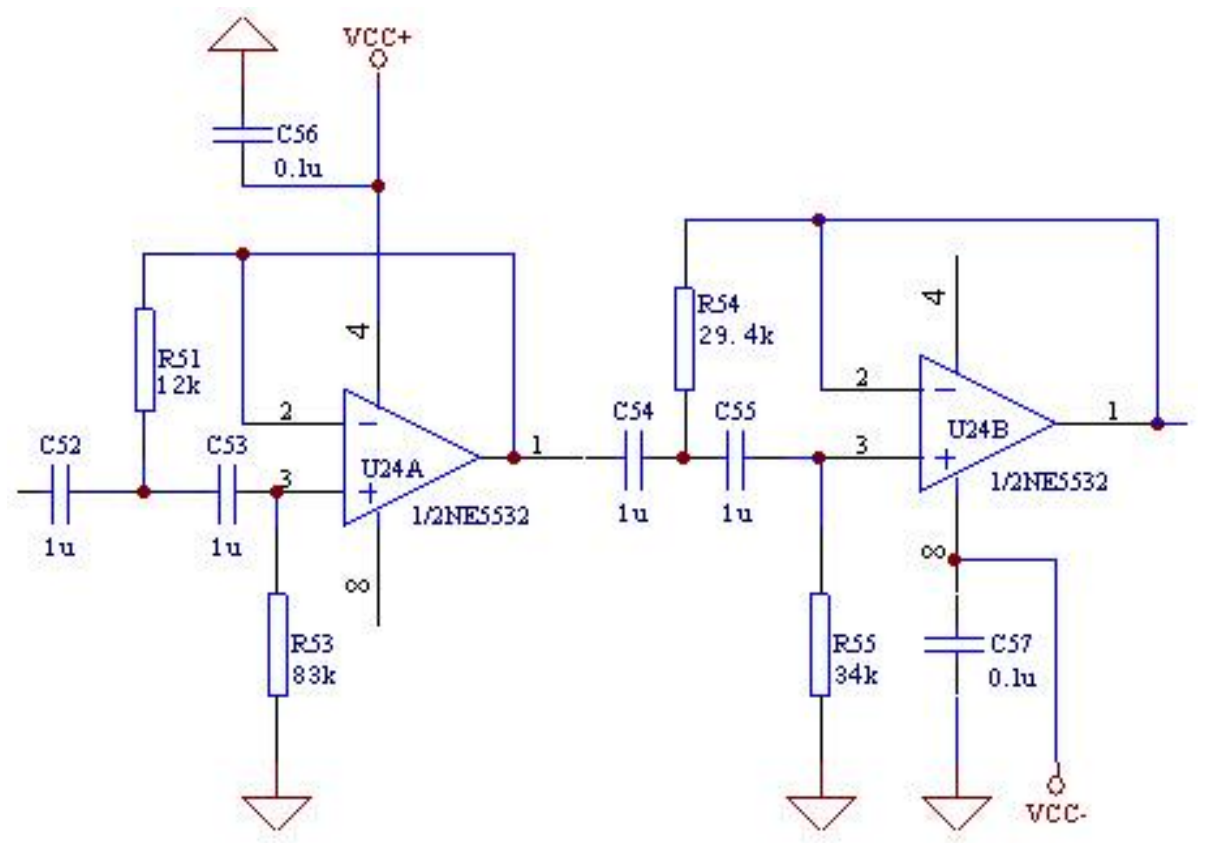

Figure 4. High-pass Filter Circuit Diagram

The transfer functions of the filter circuit: 


$$
H(s)=\frac{1}{\left(s^{2}+b_{1} s+1\right)\left(s^{2}+b_{2} s+1\right)}
$$

Where: $b_{1}=0.765, b_{2}=1.848$

If you select the capacitance values satisfy: $b_{1}=\frac{2}{C_{1}}, \quad 1=\frac{1}{C_{1} C_{2}}, \quad b_{2}=\frac{1}{C_{3}}$, $1=\frac{1}{C_{3} C_{4}}$, That $\mathrm{C} 42=2.61 \mathrm{~F}, \mathrm{C} 43=0.38 \mathrm{~F}, \mathrm{C} 44=1.08 \mathrm{~F}, \mathrm{C} 45=0.924 \mathrm{~F}$, select $\mathrm{R} 42$ $=\mathrm{R} 43=\mathrm{R} 44=\mathrm{R} 45=1 \Omega$ constitutes a cutoff frequency of $1 \mathrm{rad} / \mathrm{s}$ fourth-order Butterworth filter. Take the scale factor $k_{f}=\frac{\omega_{o}^{\prime}}{\omega_{o}}=\frac{2 \pi \cdot 5000}{1}=31415.9$, Moved to make the cutoff frequency $5 \mathrm{kHz}$, Take amplitude scaling factor $k_{m}=10000$,You can use $10 \mathrm{k} \Omega$, rather than $1 \Omega$ resistance, whereby the proportion of component value: $\mathrm{R} 42=\mathrm{R} 43=\mathrm{R} 44=\mathrm{R} 45=10 \Omega, \mathrm{C} 42=8300 \mathrm{pF}, \mathrm{C} 43$ $=1200 \mathrm{pF}, \mathrm{C} 44=3400 \mathrm{pF}, \mathrm{C} 45=2900 \mathrm{pF}$. Similarly, the ratio method using high-pass filter component values shown in Fig. 4 can be obtained. Experimental know the band-pass filter pass band frequency range of about $4.82 \mathrm{~Hz} \sim 4.91 \mathrm{kHz}$, will not result in the loss of heart sound frequency components. ECG and heart sound processing circuit similar to the circuit, only the band-pass filter pass-band frequency range of about $0.03 \sim 160 \mathrm{~Hz}$ [18-19].

\subsection{Hz Notch Circuit}

Frequency interference is a major interference ECG heart sounds, although the preamplifier circuit for common mode interference has a strong inhibitory effect, but some are differential mode interference into the circuit, and the frequency in the frequency range of ECG heart sounds inside, so that the ECG heart sound output stage circuit before there is a big frequency interference, it must be specially filtered out. As shown in Figure 5. R61, R62, R63, C61, C62, C63, U25A, U25B

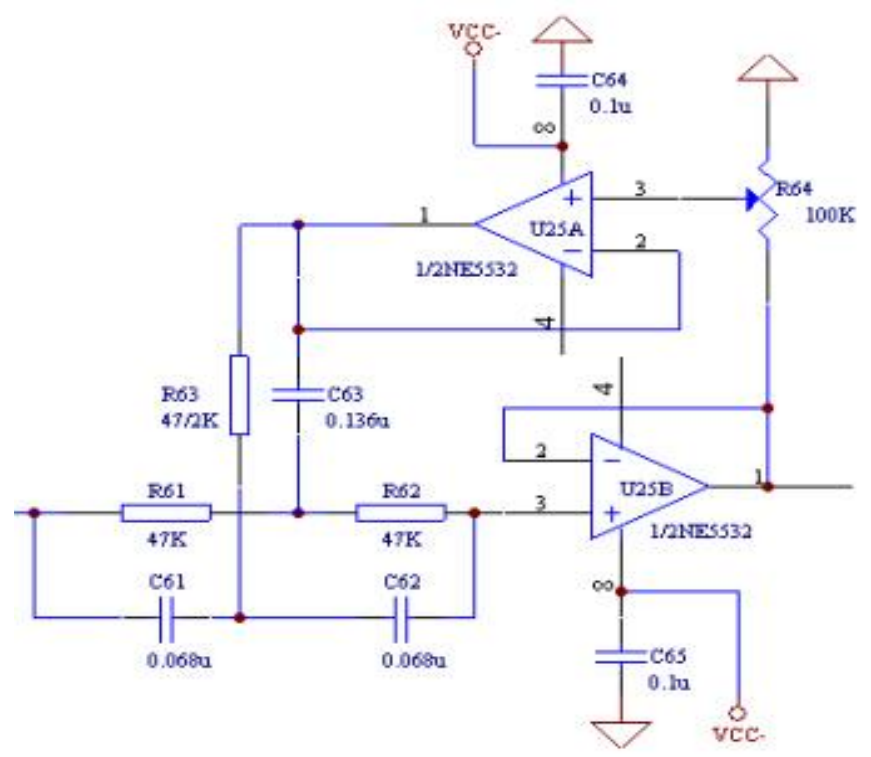

Figure 5. 50Hz Notch Circuit 
Constitute a $50 \mathrm{~Hz}$ notch circuit, choose $\mathrm{C}=0.068 \mu \mathrm{F}$, then the resistance $\mathrm{R}$ by:

$$
f_{o}=1 / 2 \pi R C=50 \mathrm{~Hz}
$$

calculated $\mathrm{R}=47 \mathrm{k} \Omega$, the experiment debugging, the circuit center frequency between $49.9 \mathrm{~Hz} \sim 50 \mathrm{H}$, notch depth of $80 \mathrm{~dB}, 3 \mathrm{~dB}$ bandwidth of about $1.7 \mathrm{~Hz}$, the notch effect is ideal [20].

\subsection{Optical Isolation Circuit}

In consideration of the acquisition process, patient safety and outside electrical interference, the circuit between a strong signal (220V AC) with weak signal (ECG heart sounds) are using optical isolation technology. Use DCP010512DP 5V input DC / DC converter power supply circuit section for signal amplification, isolated strong signal in direct contact with the human body. Use chip ISO175P cut signal amplification circuit and subsequent circuit direct contact, with the optical signal transmission to achieve the isolation of weak signals [20]. A typical chip wiring diagram is shown below in Figures 6 and 7.

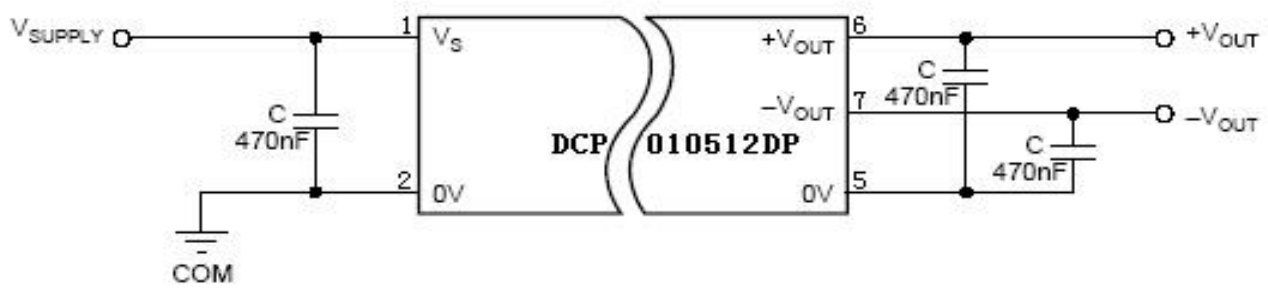

Figure 6. DCP010512DP Typical Wiring Diagram

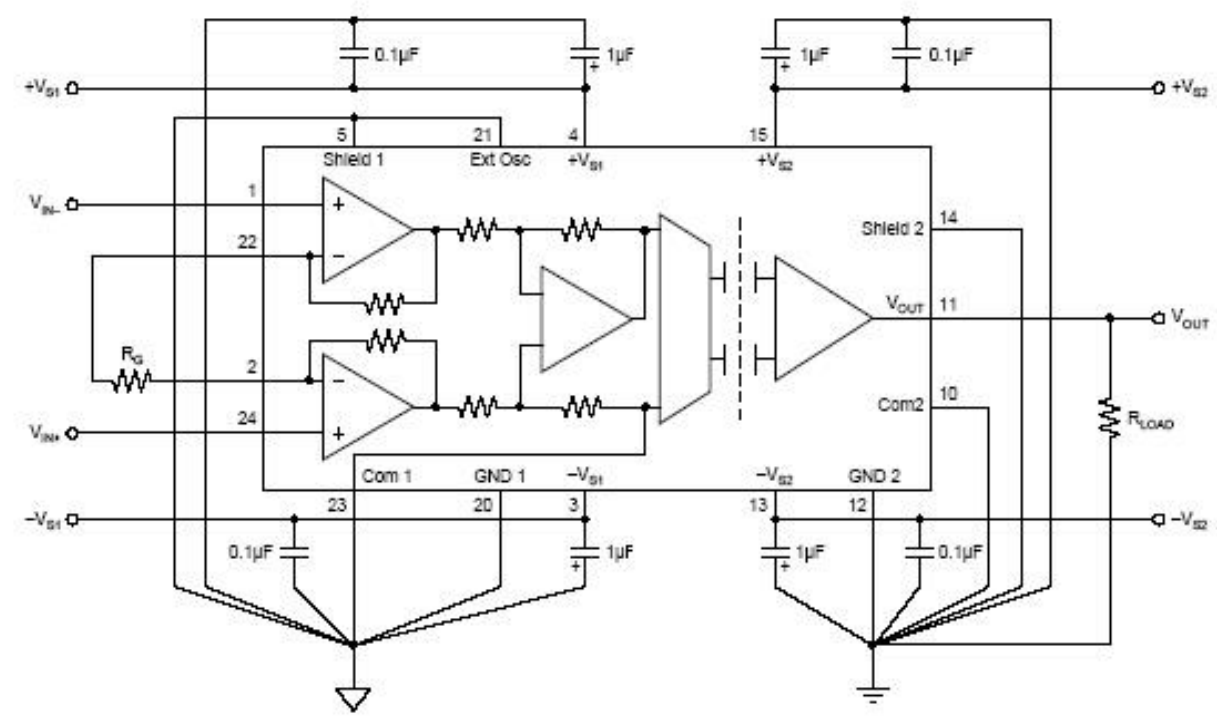

Figure 7. ISO175P Typical Wiring Diagram

\subsection{PCI - 9101 Data Acquisition Card}

PCI - 9101 board is PCI bus data acquisition board, the board can be directly inserted into the PCI slot of industrial computer or personal computer, a analog voltage signal, digital voltage signal acquisition and analog signals, digital output voltage signal output system. PCI - 9101 board provides users with single/double 
eight road 16 analog input data acquisition channel, a 12 bit analog signals output, 8 bit TTL digital quantity input and 8 bit TTL digital output [21].

\section{Software Design}

Heart sounds ECG real-time auscultation system based on LabVIEW is mainly composed of sensors, signal conditioning circuit, PCI - 6023 data acquisition card and computer four parts. Among them, the heart sound sensor and ECG sensor is to obtain the effect of the human body heart sounds ECG signal, translates into electrical signal, the signal is generally weak, accompanied by noise, should be based on the modulation circuit filter and amplification and other processing. Data acquisition card for sampling, these signals into digital signal after into the computer to display and save [22-23].

For the quality of the sensor and the signal conditioning is not here. Software mainly include automatic display, storage, and signal playback and analysis of the two big modules. LabVIEW2011 acquisition software based on virtual instrument development platform for development, it mainly controls the A/D data acquisition card, and the output after signal conditioning of the analog signal into digital signal of computer can handle, in the automatic display and storage, the user only for file storage location, the buffer data volume and the parameters of the sampling frequency, the software can display after the data storing data in real time to the designated location. These storage file can also be in signal playback and analysis module for playback and simple analysis.

\subsection{Program Flow Chart}

The program design flow chart shown in Figure 8, Set the data directory first, then to set of acquisition parameters. If the environment is noisy, still can set the filter parameters. Program according to the data buffer size to judge whether the data acquisition end, if the data buffer is 0 , end of data collection.

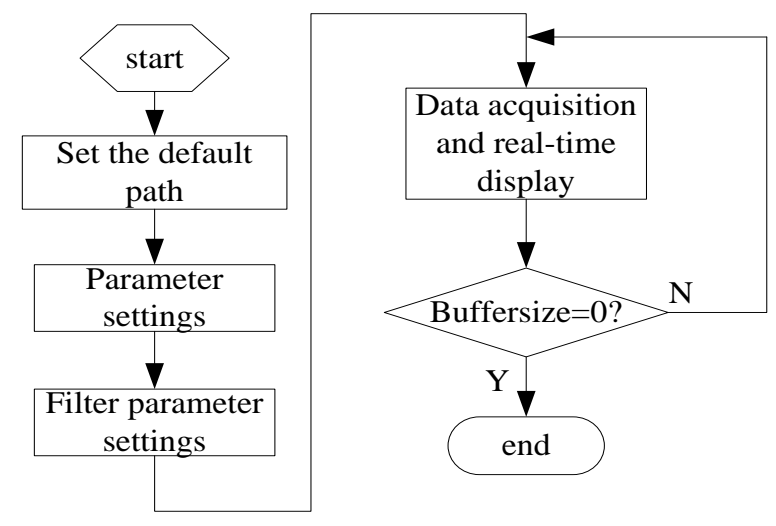

Figure 8. Program Flow Chart

\subsection{The Front Panel Design}

Signal acquisition and real-time display the front panel as shown in Fig.9. Program consists of two main interface TAB, One is auscultation and data acquisition, the other is the playback and analysis, the graph shows the auscultation and data acquisition mainly introduced in this paper. In the main interface to set the default path is used to set data storage directory, the maximum sampling number and 
the minimum number for collection and the number of Settings, sampling frequency is used to set the data collection interval, number of buffer size and the biggest acquisition agreement.

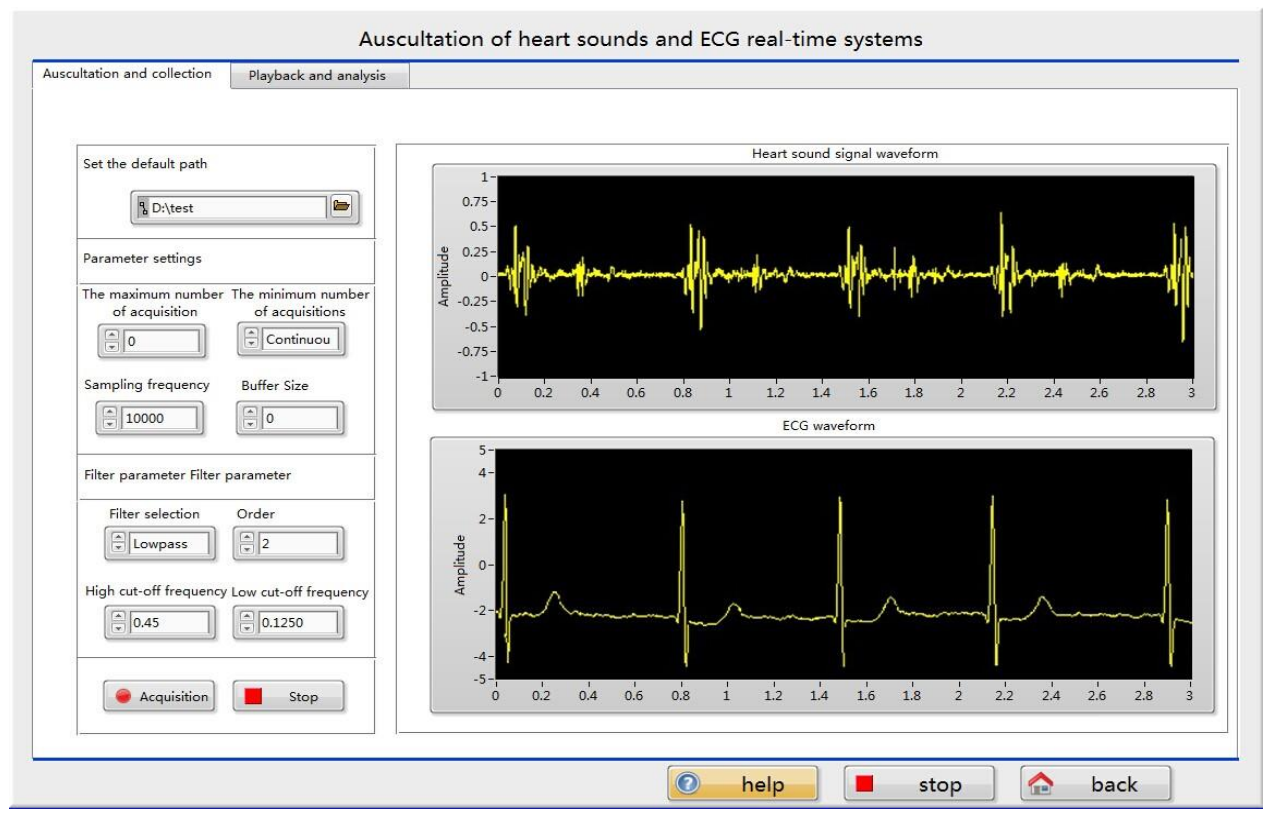

Figure 9. The Front Panel

\subsection{The Diagram Design}

Program block diagram is shown in Figure 10. First through the analog input of the VI AiConFig and DeviceNumber AiStart set A/D card and channel number, and set the sampling frequency, Then use AiRead read A/D sampling data, and stored in A matrix, and finally remove the data display in the oscilloscope.

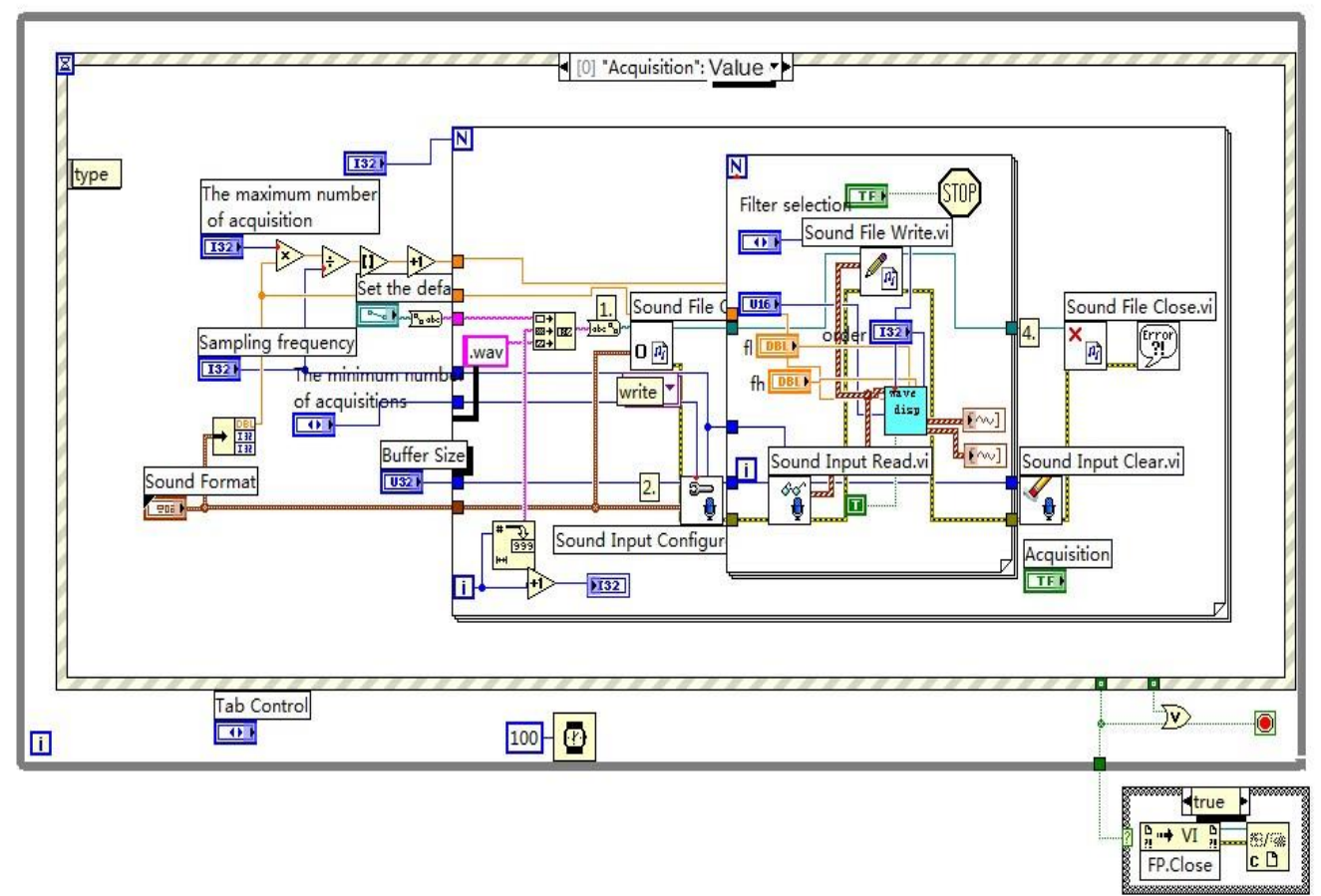

Figure 10. Program Block Diagram 


\section{Conclusion}

In this paper, the signal conditioning part use teck (Tektronix) company TDS210 monitor, the signal power amplifier is controlled by the end of the potentiometer. 50 hz notch circuit provided by the company Tektronix AFG310 signal source. Software part using virtual instrument technology, make the heart sound signal acquisition, display and analysis become very intuitive, Acquisition process is simple, the use of this system makes the heart sounds auscultation process is no longer totally dependent on the doctor's experience and knowledge. Use show that the system is stable and reliable, and the result is very ideal.

\section{References}

[1] K. Grau, I. Tetens, K. S. Bjørnsbo and B. L. Heitman, „Overall glycaemic index and glycaemic load of habitual diet and risk of heart disease", Public Health Nutrition, vol. 14, no. 1, (2010), pp.109-18.

[2] T. v. D. Bom, A C. Zomer, A. H. Z winderman, F. J. M eijboom, B. J. Bouma and B. J M Bulder, „The changing epidemiology of congenital heart disease“, Nature Reviews Cardiology, vol. 8, no. 1, (2010), pp. 50-60.

[3] P. K. Myint, R. N. Luben, P. G. Surtees, N. W. J. Wainwright, N. J. Wareham and Khaw Kay-Tee, Physical functional health predicts the incidence of coronary heart disease in the European Prospective Investigation into Cancer-Norfolk prospective population-based study", International Journal of Epidemiology, vol. 39, no. 4, (2010),pp. 996-1003

[4] X.-y. Yang, X.-f. Li, X.-d. Lü and Y.-l. Liu, „Incidence of congenital heart disease in Beijing“, China, Chinese Medical Journal, vol. 122, no. 10, (2009), pp.1128-32.

[5] I. Maglogiannis, E. Loukis, E. Zafiropoulos and A. Stasis, „Support Vectors Machine-based identification of heart valve diseases using heart sounds", Computer Methods and Programs in Biomedicine, vol. 95, no. 1, (2009), pp. 47-61

[6] D. A. Kelmenson, J. K. Heath, S. A. Ball, H. M. A. Kaafarani, E. M. Baker, D. D.Yeh, E. A. Bittner, M. Eikermann and J. Lee, „Prototype electronic stethoscope vs conventional stethoscope for auscultation of heart sounds“", Journal of Medical Engineering \& Technology, vol. 38, no. 6, (2014), pp. 307-310.

[7] J. Z. Luo and L. Luo, „Cardiac auscultation“, Edited by People's medical publishing house Press Publishing (2001).

[8] J. R. Zhang, C. Wang and G. Y. Zhao, ,Proficient in LabVIEW virtual instrument program design and case implementation", Edited by People's posts and telecommunications Press Publishing, Beijing (2013).

[9] H. X. Hong, X. L. You and Y. M. Zou, „Labview8.0 portal and improve case tutorial“, Edited by China petroleum \& chemical corporation Press Publishing, Beijing (2010).

[10] H. Tang, T. Li, Y. Park and T. Qiu, „Separation of heart sound signal from noise in joint cycle frequency-time-frequency domains based on fuzzy detection“, IEEE Transactions on Biomedical Engineering, vol. 57, no. 10, (2010), pp. 2438-47.

[11] A. Mondal, P. S. Bhattacharya and G. Saha, ,Reduction of heart sound interference from lung sound signals using empirical mode decomposition technique“, Journal of Medical Engineering \& Technology, vol. 35, nos. 6-7, (2011), pp. 344-353.

[12] Y. Li, C. C. Y. Poon and Y.-T. Zhang, ,Analog integrated circuits design for processing physiological signals“, IEEE Reviews in Biomedical Engineering, vol. 3, (2010), pp. 93-105.

[13] F. Liu, Y. Wang and Y. Wang, „Research and Implementation of Heart Sound Denoising“, Physics Procedia, vol. 25, (2012), pp. 777-785.

[14] S. Karpagachelvi, M. Arthanari and M. Sivakumar, „ECG Feature Extraction Techniques - A Survey Approach“, International Journal of Computer Science and Information Security, vol. 8, no. 1, (2010), pp.76.

[15] C. L. Dong, S. Q. Tao and Y. Z. Cheng, „Practical Cardiology“, Edited by Shanghai Science and Technology Press Publishing, Shanghai (2010).

[16] S. Franco, „Design With Operational Amplifiers And Analog Integrated Cicuits“, Edited by Xi'an Jiaotong University Press Publishing (2009).

[17] Information on: http://www.analog.com,2014

[18] J. W. Nilsson and L. Q. Xi, „Electric Circuits sixth Edition“, Edited by Electronic Industry Press Publishing,Beijing (2002).

[19] S. B. Tong, „Analog Electronics 2nd Edition“, Edited by Higher Education Press Publishing,Beijing (1988).

[20] Information on:http://www.ti.com, (2014).

[21] Information on: http://www.amzc.com.cn/productshow.asp?articleid=243, (2014) 
[22] S. X. Chen and X. Liu, „Labview bible“, Edited by The electronics industry Press Publishing, Beijing (2011).

[23] Z. Yang, Y. Yuan and Y. J. Jia, „Virtual instrument is introductory tutorial teaching experiment“, Edited by Beijing aerospace university Press Publishing,Beijing (2008).

\section{Author}

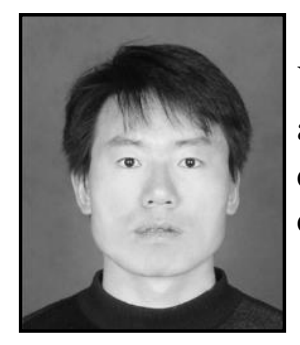

Kunliang Xu, He received his M.Sc. in Computer (2006) from Yunnan University. Since 2006 he is a teacher of Computer Science and Engineering department of Qujing Normal University. He is current research interests include different aspects of Electronic circuit design, computer software and virtual instruments. 